\title{
Cisti parapieliche: un elemento di sospetto per la diagnosi di Malattia di Fabry
}

\author{
Livia Maria Sorrentino, Luigi Annicchiarico Petruzzelli, Angela Maria Pellegrino, Cristina Marchetiello, Maria Amicone, \\ Eleonora Riccio, Antonio Pisani
}

Cattedra di Nefrologia, Dipartimento di Sanità Pubblica, Università degli Studi di Napoli Federico II, Napoli

\section{Parapelvic cysts: a suspicious feature of Fabry disease}

Although parapelvic cysts are not considered the cardinal renal manifestation of Fabry disease and are reported in few cases in the literature, their finding in Fabry disease is very frequent. Therefore in their presence, as showed in this case, a diagnosis of Fabry disease should always be considered.

Keywords: Fabry's disease, Parapelvic cysts, Renal ultrasound

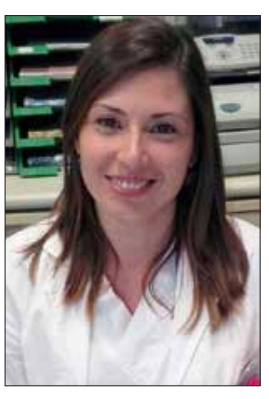

Livia Maria Sorrentino

\section{Caso clinico}

Un uomo di 63 anni si ricovera presso l'Unità di Cardiologia per essere sottoposto a impianto di defibrillatore. Durante l'ospedalizzazione si ha per la prima volta riscontro di alterazione degli indici di funzione renale, con diagnosi di insufficienza renale cronica (IRC) III stadio e proteinuria $500 \mathrm{mg} / 24 \mathrm{~h}$ e pertanto viene riferito alla valutazione specialistica nefrologica.

Dall'anamnesi patologica remota non si nota nulla di rilevante, eccetto la presenza, già a valutazioni ecografiche renali precedenti, di 2 cisti parapieliche bilaterali (Figg. 1A, B). Benché il paziente non avesse manifestazioni o storia familiare suggestive per una malattia ereditaria, l'osservazione di cisti parapieliche associate a insufficienza renale cronica, proteinuria ed eventi cardiovascolari deve sempre evocare la diagnosi di Malattia di Fabry (FD). Per questo vengono praticati sia il dosaggio dei livelli di alfa-galattosidasi $A$

Accepted: April 7, 2017

Published online: April 21, 2017

Indirizzo per la corrispondenza:

Dr.ssa Livia Maria Sorrentino

Cattedra di Nefrologia

Dipartimento di Sanità Pubblica

Università degli Studi di Napoli Federico II

Via Pansini 5

80131 Napoli

liviasorrentino@hotmail.com
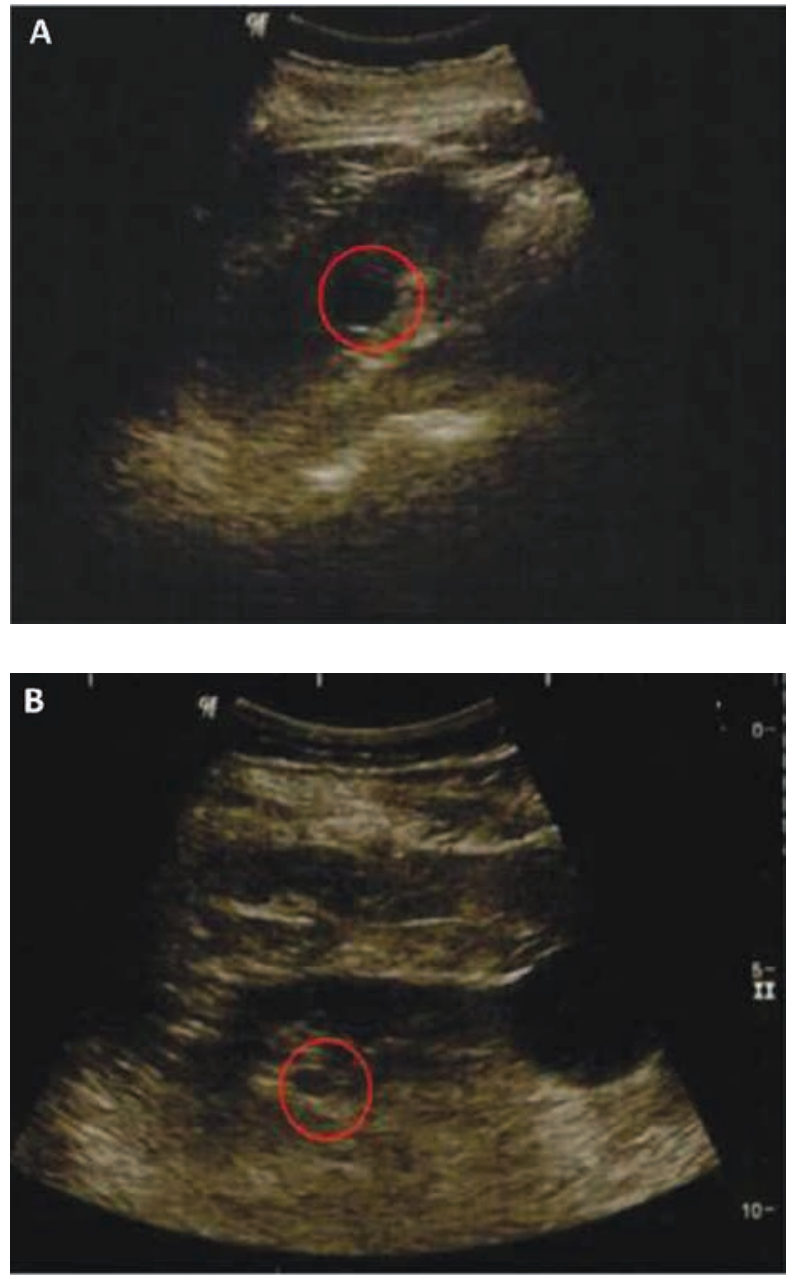

Figg. 1A, B - Immagini ecografiche di cisti parapieliche nelle scansioni coronali del rene di destra (A) e di sinistra (B). 
che l'analisi genetica, esami che confermano la diagnosi. II paziente presenta infatti bassi livelli di alfa galattosidasi $\mathrm{A}$ (0.2 nmol $4 \mathrm{MU} / \mathrm{mL} / \mathrm{h}$; valori normali 8-19 nmol $4 \mathrm{MU} / \mathrm{mL} / \mathrm{h}$ e l'analisi della sequenza del gene GLA mostra una mutazione puntiforme in omozigosi al nucleotide c.901 C>T nell'esone 6 (p.Arg301X).

Anche se le cisti parapieliche non sono quindi considerate una manifestazione renale patognomonica della FD (1) e ne sono riportati solo pochi casi in letteratura $(2,3)$, il loro riscontro nei pazienti affetti è molto frequente (4). Pertanto, quando sono presenti, specialmente in associazione con altri sintomi/segni facenti parte del corteo della FD, la diagnosi di Malattia di Fabry deve essere sempre presa in considerazione.

\section{Disclosures}

Financial support: No financial support was received for this submission. Conflict of interest: The authors have no conflict of interest.

\section{Bibliografia}

1. Pisani A, Visciano B, Imbriaco M, et al. The kidney in Fabry's disease. Clin Genet. 2014;86(4):301-309.

2. Sayer JA, Haslam P, Brennan P. Parapelvic cysts leading to a diagnosis of Fabry disease. Kidney Int. 2008;74(1):1366.

3. Pisani A, Riccio E, Cianciaruso B, Imbriaco M. Simultaneous multicystic kidney and Anderson-Fabry disease: 2 separate entities or same side of the coin. J Nephrol. 2011;24(6):806-808.

4. Ries M, Bove Bettis KE, Choyke P, et al. Parapelvic kidney cysts: a distinguishing feature with high prevalence in Fabry disease. Kidney Int. 2004;66(3):978-982.

\section{TEST DI VERIFICA}

\section{La trasmissione genetica nella Malattia di Fabry è:}
a) Legata al cromosoma $X$
b) Autosomica dominante
c) Autosomica recessiva.

2. Gli organi più frequentemente coinvolti nelle manifestazioni tardive della Malattia di Fabry sono:
a) Occhio e cute
b) Encefalo, rene, cuore
c) Apparato gastrointestinale e scheletrico.

3. La Terapia Enzimatica Sostitutiva (ERT) nella Malattia di Fabry viene iniziata:

a) In tutti i pazienti al momento della diagnosi, indipendentemente dalle manifestazioni cliniche b) Solo nelle diagnosi in età pediatrica

c) Nei maschi adulti al momento della diagnosi, nei ragazzi allo sviluppo di sintomi significativi e nelle femmine allo sviluppo di sintomi/segni indicativi di danno d'organo.

4. Perché il riscontro di cisti parapieliche deve far sospettare la diagnosi di Malattia di Fabry?
a) Per la loro presenza più comune e più precoce nei pazienti affetti rispetto alla popolazione generale
b) Perché rappresentano una manifestazione renale caratteristica della malattia
c) Perché possono provocare una sintomatologia rilevante.

